\title{
Unexpected laboratory results in cold agglutinin disease
}

\author{
Nesibe Esra Yasar, Aysegul Ozgenc, Ibrahim Murat Bolayirli, Mutlu Adiguzel, Dildar Konukoglu \\ Department of Medical Biochemistry, Medical Faculty of Cerrahpasa, Istanbul University, Istanbul, Turkey
}

\begin{abstract}
Cold agglutinin disease (CAD) is an autoimmune hemolytic disease in which antibodies for erythrocyte surface antigens are activated by low temperatures, causing agglutination of erythrocytes. Most of the published cases in the literature are related to the clinical presentation of the disease. Cold agglutinins can also interfere with laboratory tests. In this case, unexpected haptoglobin ( $\mathrm{Hp}$ ) and glycated hemoglobin ( $\mathrm{HbA1c}$ ) results, as well as complete blood count $(\mathrm{CBC})$, were reported. A 55-year-old woman with joint paint presented the rheumatology polyclinic. Blood samples were collected into anticoagulated and gel separation tubes for $\mathrm{CBC}, \mathrm{HbA} 1 \mathrm{c}$, and biochemistry tests. The automated hematology analyzer results indicated a very low hematocrit (7\%) and erythrocyte count $\left(0.57 \times 10^{6} \mathrm{~mm}^{3}\right)$. The erythrocyte indices, platelet count, and mean platelet volume could not be measured. Hp was undetectable. The HbA1c value was below the reference threshold. The patient's file revealed that she had been diagnosed with CAD 5 years earlier. The samples were reanalyzed after warming in a Benmarie for 25 minutes to $37^{\circ} \mathrm{C}$. While the $\mathrm{CBC}$ results improved, the $\mathrm{HbA} 1 \mathrm{c}$ and $\mathrm{Hp}$ results did not change. Informing the laboratory when samples to be used are from patients with $\mathrm{CAD}$ and providing the proper temperature conditions during transportation will ensure accurate results, as well as decrease workload and laboratory costs.
\end{abstract}

Keywords: Cold agglutinin disease, complete blood count, interference

C old agglutinin disease (CAD) is an autoimmune hemolytic disease in which the antibodies for erythrocyte surface antigens are activated by low temperatures, causing agglutination of red blood cells (RBC). Autoantibodies may be idiopathic or secondary to infection, malignancy, and other autoimmune diseases. CAD is a rare disease and accounts for $15 \%$ of all autoimmune hemolytic anemias. The incidence of CAD is 1 per million people per year [1]. Most of the published cases in the literature are related to the clinical presentation of disease. Cold agglutinins can also interfere with laboratory tests. Several case studies have reported that cold antibodies caused erroneous laboratory results, especially in the complete blood count (CBC) and blood group test $[2,3]$.

In the present case, in addition to the $C B C$, unexpected haptoglobin $(\mathrm{Hp})$ and glycated hemoglobin $(\mathrm{HbA} 1 \mathrm{c})$ results were reported.

\section{Case Report}

A 55-year-old woman with joint paint presented at the rheumatology polyclinic. She had a history of chronic obstructive pulmonary disease, rheumatoid arthritis, hypertension, and hyperthyroidism. Blood samples were collected into anticoagulated (K3 EDTA, 2 mL, 367836; BD Life Sciences-Diagnostic Systems, Franklin Lakes, NJ, USA) and gel separation tubes (SST II, 5 mL, 367953; BD Life Sciences-Diagnostic Systems, Franklin Lakes, NJ, USA) for CBC, HbA1c, and biochemistry tests.

\section{Results}

The automated hematology analyzer (Unicel DxH 800; Beckman Coulter, Inc., Brea, CA, USA) results were a very low hematocrit $(7 \%)$ and $\mathrm{RBC}$ count $\left(0.57 \times 10^{6} \mathrm{~mm}^{3}\right)$. Mean corpuscular 
Table 1. Comparative results of samples at room temperature (A) and after warming to $37^{\circ} \mathrm{C}$ (B)

\begin{tabular}{|c|c|c|c|}
\hline Parameters & A & B & Reference range \\
\hline Hematocrit (\%) & 7 & 30 & $36-48$ \\
\hline $\mathrm{MCV}(\mathrm{fL})$ & 123 & 101.3 & $80-99$ \\
\hline $\mathrm{MCH}(\mathrm{pg})$ & Unmeasured & 31.4 & $27.2-33.5$ \\
\hline MPV (fL) & Unmeasured & 8.9 & $6.9-10.8$ \\
\hline HbA1c (\%) & 3.6 & 3.6 & $4.8-6$ \\
\hline Glucose (mg/dL) & 102 & & $76-110$ \\
\hline $\mathrm{Hp}(\mathrm{mg} / \mathrm{dL})$ & Undetected & Undetected & $30-200$ \\
\hline $\mathrm{LDH}(\mathrm{IU} / \mathrm{L})$ & 232 & & $<250$ \\
\hline Hemolysis index & 12 & & \\
\hline
\end{tabular}

AST: Aspartate aminotransferase, HbA1c: Glycated hemoglobin, Hp: Haptoglobin, LDH: Lactate dehydrogenase, MCH: Mean corpuscular hemoglobin, MCHC: Mean corpuscular hemoglobin concentration, MCV: Mean corpuscular volume, MPV: Mean platelet volume, PLT: Platelet, RBC: Red blood cell.

volume (MCV), mean corpuscular hemoglobin $(\mathrm{MCH})$, mean corpuscular hemoglobin concentration (MCHC), platelet (PLT) count and mean platelet volume (MPV) could not be measured. Overt agglutination was observed when the patient's test tubes with EDTA were examined (Fig. 1). The patient's file revealed that she had previously had hemoglobin-hematocrit incompatibility and that her blood group couldn't be determined in preoperative examinations before a cholecystectomy. She had been diagnosed with CAD 5 years earlier based on an increased cold agglutinin titer (1:56) and decreased C3 and $C 4$ levels.

As CAD is characterized by in vivo hemolysis, lactate dehydrogenase (LDH), aspartate aminotransferase (AST), total-direct bilirubin and $\mathrm{Hp}$ were determined as the markers of hemolysis (Cobas c702; Roche Diagnostics, Basel, Switzerland). While all other markers were within the reference ranges, $\mathrm{Hp}$ was undetectable (Table 1).

The patient's HbA1c value (Variant II Turbo, Bio-Rad Laboratories, Inc., Hercules, CA, USA) was below the reference threshold (3.6\%), while the glucose level was $102 \mathrm{mg} / \mathrm{dL}$. Given the interference caused by cold agglutinins, the samples were reanalyzed after warming in a benmarie for 25 minutes to $37^{\circ} \mathrm{C}$. While $\mathrm{CBC}$ results improved, the $\mathrm{HbA} 1 \mathrm{c}$ and $\mathrm{Hp}$ results did not change (Table 1). Hemoglobin variant analysis was performed to investigate the cause of the low HbA1c value (Primus Ultra; Trinity Biotech, Plc, Bray, Ireland) and no abnormal peak was detected.

\section{Discussion}

Cold agglutinins are antibodies directed against I antigen on the surface of erythrocytes. These are usually IgM types, and rarely, IgG and IgA types of antibodies. These antibodies are activated by cold temperature and bind to the surface of the RBC. The resulting antigen antibody complex activates the complement cascade, usually resulting in extravascular hemolysis, and rarely, intravascular hemolysis [1].

Cold agglutinins may also be found in low titers of the sera of healthy individuals, but they show no activity above $4^{\circ} \mathrm{C}$. Pathological cold agglutinins usually react at $28^{\circ} \mathrm{C}$ to $31^{\circ} \mathrm{C}$, or even up to $37^{\circ} \mathrm{C}$. The highest temperature at which the antibodies continue to be activated is called the thermal amplitude [4]. Clinical presentation and the severity of laboratory findings depend on the degree of thermal amplitude, rather than the antibody titer [1].

Cold antibodies may be monoclonal or polyclonal. Monoclonal antibodies are more commonly seen in idiopathic cases and CAD secondary to lymphoproliferative diseases. Polyclonal antibodies are generally determined after mycoplasma pneumonia, Epstein-Barr virus, or cytomegalovirus infections [2].

Circulatory symptoms, such as hemolytic anemia, Raynaud's disease, livedo reticularis, acrocyanosis, and rarely, cutaneous necrosis, which are particularly aggravated during winter, can be seen in CAD [1]. However, the first suspicion of CAD is usually a result of a laboratory's inability to measure RBC count and related indices [4]. For CBC analyzers, cell counts are based on cell diameters. In CAD, antibodies are activated at low temperatures and lead to degeneration and autoagglutination of the erythrocyte membrane. The resulting micro-aggregates can be counted as leukocytes or single erythrocytes. Very large aggregates may not be evaluated. All of these lead to an erroneously low RBC count. Since hematocrit and erythrocyte indices are also calculated using RBC counts, errors are also seen in these 


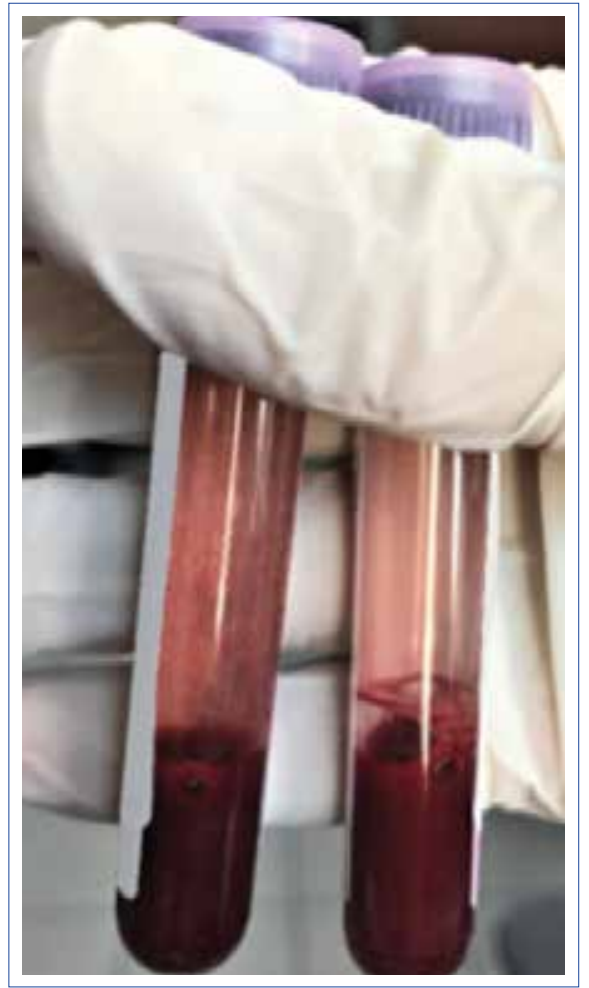

Figure 1. Anticoagulated blood sample of the patient with spontaneous agglutination on left and normal sample on the right

parameters. The hematocrit will be spuriously low. MCV, $\mathrm{MCH}$ and $\mathrm{MCHC}$ values have also been found to be elevated or not measurable. Since hemoglobin is measured directly by the lysis of erythrocytes in another channel, it is not affected by CAD [2]. PLT counts and indices can also be measured incorrectly, as platelets can also undergo autoagglutination. Another laboratory test that cold agglutinins may hinder is blood group determination. The way to avoid all of these unsuccessful analysis attempts is to transfer the sample at $37^{\circ} \mathrm{C}$ or warm the sample to $37^{\circ} \mathrm{C}$ before analysis [4]. In our case, the RBC count and the hematocrit did not correlate with the hemoglobin, and erythrocyte indices indicated that the MCV was high, and the $\mathrm{MCH}$ and MCHC could not be measured. The PLT count and MPV were also not measurable. These values improved after the sample was warmed to $37^{\circ} \mathrm{C}$. In a case report published by Nikousefat et al., [5] a 72-year-old female patient was found to have a low RBC count, incompatible hematocrit and hemoglobin, and increased erythrocyte indices. They evaluated the cold agglutinin antibody titer and the patient was diagnosed with CAD. The laboratory values were improved by heating the sample to $37^{\circ} \mathrm{C}$. Kalyani et al. [4] reported of a 45 -year-old male patient who had cold agglutinin, a decreased RBC count, and increased MCV and MCHC. Kumar et al. [6] described severe thrombocytopenia incompatible with the clinical status of a 35-year-old female patient due to cold agglutinin interference. In a case report submitted by Lodi et al., [7] the blood group of a 48-year-old male patient was not determined as a result of cold agglutinins and the patient died as a result of compli- cations due to the emergency transfusion of universal RBCs ( 0 Rh-positive).

$\mathrm{Hp}$ is a tetrameric protein consisting of 2 light chains (alpha) and 2 heavy chains (beta) and is synthesized primarily in hepatocytes in response to the secretion of cytokines like interleukin (IL)-6, IL-1, and tumor necrosis factor. Hp is characterized by molecular heterogeneity due to genetic polymorphism. Three main phenotypes have been identified: $\mathrm{Hp} 1-1, \mathrm{Hp} 2-1$ and Hp22. Another phenotype, $\mathrm{HpO}$, causes the absence or a reduced level of $\mathrm{Hp}$ in plasma (referred to as ahaptoglobinemia and hypohaptoglobinemia, respectively). The main function of $\mathrm{Hp}$ in the organism is to bind free hemoglobin released from the intravascular destruction of erythrocytes [8]. In our case, the concentration of serum $\mathrm{Hp}$ was undetectable. However, there was no severe anemia in the patient and the hemolysis markers of $\mathrm{LDH}, \mathrm{AST}$, total and direct bilirubins were in the reference range. A low serum $\mathrm{Hp}$ value is noteworthy. We thought that cold agglutinins may have interfered analytically with the $\mathrm{Hp}$ measurement, but we did not find such information in a literature review. In addition, when the sample tube was warmed to $37^{\circ} \mathrm{C}$, the $\mathrm{Hp}$ result did not change. A low $\mathrm{Hp}$ level may be the result of in vitro hemolysis interference, but this possibility was also excluded because the hemolysis index was low in this patient. The other possible reason may be that the patient had a phenotype variant of $\mathrm{Hp}$ that the method used cannot identify. Studies have shown that the measurement of some phenotypes of $\mathrm{Hp}$ by immuno-turbidimetric methods may lead to incorrect results [9]. The previous low $\mathrm{Hp}$ level laboratory result of the patient, in spite of a moderate level of anemia and normal hemolysis markers, strengthens this possibility.

Another significant finding of this patient was the low $\mathrm{HbA} 1 \mathrm{C}$ value. According to previous and current glucose levels, the patient was considered to have impaired fasting glycemia. The reasons for interference in $\mathrm{HbA} 1 \mathrm{c}$ measurement may be a shortened red blood cell lifespan, the presence of variant hemoglobin, uremia, or drugs $[10,11]$. There was no evidence of active hemolysis or uremia in this patient. Hemoglobin variant analysis showed no abnormal peak. Since the drugs used by the patient could be queried, the possibility of adverse drug effects couldn't be ruled out.

\section{Conclusion}

Cold agglutinins can cause interference in laboratory tests. Informing the laboratory of samples from patients with CAD and ensuring the proper temperature conditions during transportation will lead to accurate results as well as decrease workload and laboratory costs.

Acknowledgements: The authors wish to thank Özlem Yalçın and Leyla Duruk for their technical assistance.

Conflict of interest: None declared.

Peer-review: Externally peer-reviewed.

Authorship contributions: Concept - N.E.Y., A.Ö.; Design - N.E.Y., 
A.Ö., M.A.; Supervision - N.E.Y., A.Ö., M.A.; Fundings - İ.M.B., D.K.; Materials - N.E.Y., A.Ö.; Data collection \&/or processing - N.E.Y., A.Ö., M.A.; Analysis and/or interpretation - I.M.B., D.K.; Literature search - D.K.; Writing - N.E.Y., A.Ö., İ.M.B.; Critical review - I.M.B.

\section{References}

1. Swiecicki PL, Hegerova LT, Gertz MA. Cold agglutinin disease. Blood 2013;122:1114-21. [CrossRef]

2. Ercan S, Calışkan M, Koptur E. 70-year old female patient with mismatch between hematocrit and hemoglobin values: the effects of cold agglutinin on complete blood count. Biochem Med (Zagreb) 2014;24:391-5. [CrossRef]

3. Javed R, Datta SS, Basu S, Chakrapani A. Resolution of Serologic Problems Due to Cold Agglutinins in Chronic Lymphocytic Leukemia. Indian J Hematol Blood Transfus 2016;32:2903. [CrossRef]

4. Kalyani R, Thej MJ, Thomas AK, Raveesha A. Chronic Cold Agglutinin Disease: A Case Report with Review of Literature. Journal of Clinical and Diagnostic Research 2012;6:480-2.
5. Nikousefat Z, Javdani M, Hashemnia M, Haratyan A, Jalili A. Cold Agglutinin Disease; A Laboratory Challenge. Iran Red Crescent Med J 2015;17:e18954. [CrossRef]

6. KumarTB, Bhardwaj N. Platelet cold agglutinins and thrombocytopenia: A diagnostic dilemma in the intensive care unit. J Anaesthesiol Clin Pharmacol 2014;30:89-90. [CrossRef]

7. Lodi G, Resca D, Reverberi R. Fatal cold agglutinin-induced haemolytic anaemia: a case report. J Med Case Rep 2010;4:252.

8. De Albuquerque Wobeto VP, Zaccariotto TR, de Fátima Sonati M. Polymorphism of human haptoglobin and its clinical importance. Genet Mol Biol 2008;31:602-20. [CrossRef]

9. Van Rijn HJ, van der Wilt W, Stroes JW, Schrijver J. Is the turbidimetric immunoassay of haptoglobin phenotype-dependent? Clin Biochem 1987;20:245-8. [CrossRef]

10. Cavagnolli G, Pimentel AL, Freitas PA, Gross JL, Camargo JL. Factors affecting $A 1 C$ in non-diabetic individuals: Review and meta-analysis. Clin Chim Acta 2015;445:107-14. [CrossRef]

11. Rhea JM, Molinaro R. Pathology consultation on $\mathrm{HbA}(1 \mathrm{c})$ methods and interferences. Am J Clin Pathol 2014;141:5-16. 